\title{
Adhesive energy, force and barrier height between simple metal surfaces
}

\author{
S. Ciraci, E. Tekman, M. Gökçedag \\ Department of Physics, Bilkent University, Bilkent 06533 Ankara, Turkey \\ Inder P. Batra \\ Almaden Research Center, IBM Research Division, 650 Harry Road, San Jose, CA 95120-6099, USA
}

\author{
A. Baratoff \\ Zurich Research Laboratory, IBM Research Division, 8803 Rüschlikon, Switzerland
}

Received 12 August 1991

\begin{abstract}
Using the self-consistent field pseudopotential method we calculated the adhesive energy, perpendicular and lateral forces and barrier height between two rigid A1(001) slabs modeling the sample and a blunt tip. We found that the adhesive energy and forces are site specific, and can lead to a significant corrugation in the constant force mode with negative force gradient. Lateral forces, which determine friction on the atomic scale are not simply proportional to the perpendicular force, and are typically one order of magnitude smaller. Our results confirm that perpendicular tip force and barrier height are interrelated for separations where the force gradient is positive.
\end{abstract}

\section{Introduction}

The interaction energy $E_{\mathrm{i}}$ between tip and sample and the force derived thereof are of relevance in scanning force microscopy (SFM) [1] when they show significant variations with the tip position. At large tip-sample separations $(z \simeq 7$ $10 \AA$ ) the force exerted by an atomically flat sample is van der Waals (VdW) in origin and is usually uncorrugated (one important exception may be a noncompact overlayer of easily polarizable adatoms). As $z$ is decreased the interaction energy becomes increasingly negative until the separation $z=z_{\mathrm{e}}$ corresponding to maximum adhesion. In the range $z>z_{\mathrm{e}}$ the perpendicular tip force $F_{\perp}=-\partial E_{\mathrm{i}} / \partial z$, becomes increasingly attractive, passes through a minimum and then decreases to become repulsive. For an atomically sharp tip it is expected that significantly strong lateral forces can also arise when the tip is posi- tioned off high symmetry positions. If the lateral force gradient exceeds the restoring spring constant, the tip starts to perform a stick-slip motion on the sample surface [2]. These lateral forces, which are fundamentally conservative, can thus induce hysteresis and losses via energy transfer to shear modes, resulting in an average microscopic friction force of nonconservative nature.

It is important to understand the origin of the force exerted on the tip by the sample in order to interpret the corrugation detected in SFM. For atoms far from the apex it is of $\mathrm{VdW}$ character and almost uncorrugated. On the other hand, it is better described in terms of nearly compensating ion-ion repulsion and ion-electron attraction between the nearest atoms at small and intermediate separations. For separations close to maximum adhesion ion-ion repulsion dominates the force on the tip apex [3]. At somewhat larger separation electron-ion attraction becomes dom- 
inant. Therefore, in the attractive force range with positive gradient the tip images the total charge density $[3,4]$. The interpretation of images obtained by SFM becomes difficult when the tip is blunt and not in registry with the sample surface $[5,6]$. Furthermore, in the range where significant forces are acting on the tip, the potential felt by electrons also undergoes significant sitedependent modifications with decreasing $z[3,7-$ 9]. The variation of the barrier height $\Phi_{\mathrm{h}}$ with $z$, and the formation of a mechanical contact have important implications in scanning tunneling microscopy (STM) as well [10].

A quantitative treatment of tip-sample interactions, more specifically the interaction energy, the forces acting on individual atoms and the variation of the barrier requires detailed computations. Another important but not completely understood issue is the origin of the microscopic friction between tip and sample. Even if the detailed atomic structure of a tip is usually unknown and affected by sharpening procedures and also during measurements, one can nevertheless theoretically analyze those interactions on the atomic scale to reveal their fundamental features. Our work is based on such a premise, and investigates the interactions between two simple metal slabs. In order to apply periodic boundary conditions and thus perform calculations on finite systems, we avoid questions of lattice mismatch and assume that both slabs (representing the sample and the blunt tip) are made from the same atomic layers. On the basis of self-consistent field (SCF) pseudopotential calculations we address the following issues: (i) The site-dependence of interaction energy and tip forces. (ii) The variation of the lateral forces with lateral and perpendicular motion of the tip. (iii) The correlation between perpendicular force and barrier height.

\section{Description of calculations}

Our results are extracted from standard SCF calculations with nonlocal ionic pseudopotentials [11] and a local exchange-correlation potential [12]. While the sample is taken as a 5-layer $\mathrm{Al}(001)$ slab, the blunt (flat) tip is represented by another $\mathrm{Al}(001)$ slab, the thickness of which is varied between 1 and 4 layers in order to assess the sensitivity of our results to computational restrictions. The separation between two slabs, $z$, was allowed to vary between 3 and 11 a.u. The lateral lattice constants, $R_{1}$ and $R_{2}$, and the interlayer spacing are maintained equal to the experimental equilibrium nearest-neighbor distance $d_{0}=5.4$ a.u. of bulk Al. The computations are performed in a tetragonal supercell $\left(R_{1}=R_{2}=5.4\right.$ and $R_{3}=$ 46 a.u.) in order to use periodic boundary conditions. The Bloch states of the whole system are expanded in $\sim 500$ plane waves corresponding to a cut-off $|\boldsymbol{k}+\boldsymbol{G}|^{2} \leq 8 \mathrm{Ry}$. The total energy and atomic forces are calculated in the momentum representation [13] for different lateral and perpendicular positions of the tip slab with a convergence criterion (rms deviation in potential energy) of $\sim 10^{-7} \mathrm{Ry}$. The arrangement of the supercell, the surface unit cell, and the various lateral tip positions for which computations were performed (top T, hollow $\mathrm{H}, \mathrm{C}$ and $\mathrm{M}$ sites) used in the SCF calculations are illustrated by insets in fig. 1.

The interaction energy, $E_{\mathrm{i}}(z)=E_{\mathrm{S}+\mathrm{T}}(z)-E_{\mathrm{S}}$ $-E_{\mathrm{T}}$, is extracted from the total energies of sample and tip slab together $\left(E_{\mathrm{S}+\mathrm{T}}\right)$, sample $\left(E_{\mathrm{S}}\right)$, and tip slab alone $\left(E_{\mathrm{T}}\right)$, each calculated separately in the same supercell described above. By definition $E_{\mathrm{i}}(z)<0$ indicates an attractive binding interaction. The minimum of $E_{\mathrm{i}}(z)$ at $z=z_{\mathrm{e}}$ is identified as the binding energy of the slabs $E_{\mathrm{b}}$. It is also customary to define the adhesive energy, $E_{\text {ad }}=E_{\mathrm{i}}(z) / 2$, which is the negative of the amount of work necessary to separate two semi-infinite slabs from $z$ to $\propto$. Note that the surface energy is the negative of $E_{\mathrm{i}}\left(z=z_{\mathrm{e}}\right) / 2$ calculated for two such slabs.

\section{Discussion of results}

In fig. 1a we show the variation of the interaction energies $E_{\mathrm{i}}(z)$ corresponding to a four-layer tip slab at the $\mathrm{H}$ - and $\mathrm{T}$-site. The calculated binding energies are 1.37 and $0.92 \mathrm{eV}$ per cell for the $\mathrm{H}$ - and $\mathrm{T}$-site, respectively. The binding en- 

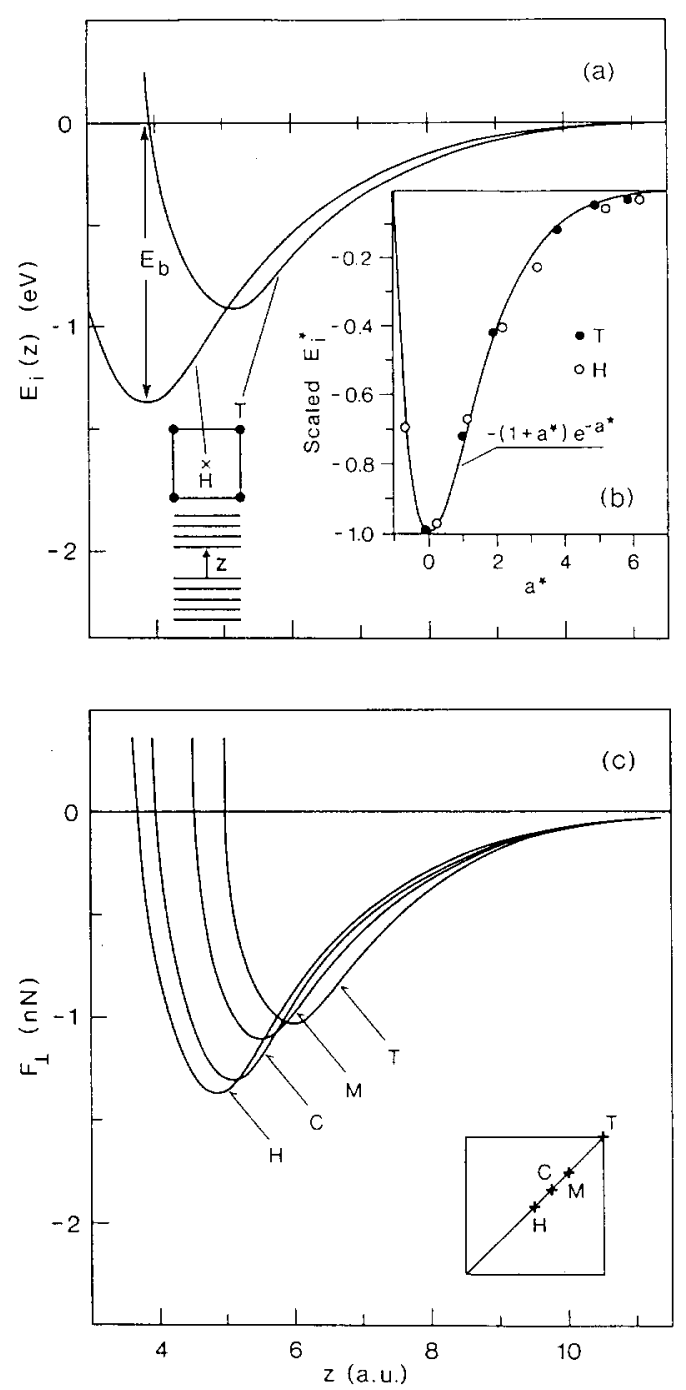

Fig. 1. (a) Interaction energy $E_{\mathrm{i}}$ versus separation $z$ between two $\mathrm{Al}(001)$ slabs at the hollow $(\mathrm{H})$ and top $(\mathrm{T})$ sites. $E_{\mathrm{b}}$ is the binding energy for maximum adhesion. (b) Scaled energy $E_{\mathrm{i}}{ }^{*}$ versus scaled separation $a^{*}$ according to the Rydberg function of Rose et al. [14]. (c) Perpendicular force on the tip slab versus separation $z$. The configuration of the two slabs with separation $z$, surface unit cell and four different relative positions (top $\mathrm{T}$, hollow $\mathrm{H}, \mathrm{C}$ and $\mathrm{M}$ sites) are shown in the insets.

ergy at the $\mathrm{H}$-site is larger since it corresponds to a natural stacking of the $\mathrm{Al}(001)$ layers in bulk Al. Note that maximum binding at the T-site occurs close to $z=d_{0}$, nearest-neighbor distance of bulk $\mathrm{Al}$. For $z>d_{0}, E_{\mathrm{i}}(z)$ at the T-site slightly exceeds that at the $\mathrm{H}$-site. The calculated interaction energies, for both sites show only small variation with the number of layers in the tip slab. For example, $E_{\mathrm{i}}$ is lowered less than $0.1 \mathrm{eV}$ in going from a single-layer to a four-layer slab at $z-z_{\mathrm{e}}$ for the T-site. However, $E_{\mathrm{i}}$ of a two-layer tip slab is $0.05 \mathrm{eV}$ lower than that of a four-layer slab. Normally, $E_{\mathrm{i}}$ is expected to decrease slightly with increasing number of layers and then to saturate. The nonmonotonic dependence of the calculated $E_{\mathrm{i}}$ is reminiscent of the oscillations of the work function and surface energy calculated earlier for thin $\mathrm{Al}(111)$ slabs [13]. These oscillations were interpreted as the manifestation of the quantum size effect due to an empty band dipping below the Fermi level as the thickness is increased.

Earlier, Rose et al. [14] proposed a simple universal relation in terms of the Rydberg function, $E_{\mathrm{i}}^{*}=-\left(1+a^{*}\right) \exp \left(-a^{*}\right)$, to scale interaction energies of flat interfaces between pairs of metals. The energy and distance are scaled by $E_{\mathrm{i}} / E_{\mathrm{b}}$ and $\left(z-z_{\mathrm{e}}\right) / \lambda$, respectively, where $\lambda$ can be taken as the screening length or a fitting parameter. This scaling of the adhesive energy has been exploited by Dürig et al. [15] to fit the variation of the force gradient with separation in a combined atomic force and tunneling $\mathrm{mi}$ croscopy measurement. The present SCF results revealing that $E_{\mathrm{i}}(z)$ is strongly site-dependent even for a simple metal interface represent a nontrivial test for the universal scaling expression. To this end we scaled the interaction energies illustrated in fig. 1a by taking $\lambda-1$ a.u. The comparison of the scaled values with the Rydberg function given in fig. $1 \mathrm{~b}$ shows good fits at both $\mathrm{H}$ - and T-sites, especially for positive force gradient. The origin of this scaling can be traced back to the form of the electron density which decays exponentially as one goes away from the surface. The inverse decay constant $\kappa$, in turn, is related to the barrier height.

The forces on individual atoms are calculated using the Hellmann-Feynman theorem. The resultant of all atomic forces in the supercell must be zero, and in mechanical equilibrium or at a local minimum all force components have to vanish. In our calculations all lattice spacings within both slabs were kept equal to the equilibrium 
bulk value. Previous calculations indicate the interlayer spacings of thin $\mathrm{Al}$ slabs deviate from the bulk value [13], and hence internal strains and forces derived thereof are indigenous to our results. The total internal forces in each slab must cancel out, but the force due to the interslab interaction remains. Accordingly, the lateral forces on each $\mathrm{Al}(001)$ layer and the net perpendicular force on each slab are physically significant. Fig. 1c illustrates the variation of the perpendicular force on the single-layer tip slab for various lateral positions. Along the line from the $\mathrm{H}$ - to the T-site, the strongest attraction occurs at the smallest $z_{\mathrm{e}}$ for the $H$-site. As the tip slab is moved from the $\mathrm{H}$ - towards the $\mathrm{T}$-site, the minimum of $F_{\perp}(z)$ gradually shifts to larger $z$ and concomitantly the magnitude of the attractive force decreases. This behavior can be explained by the decreased ion-ion repulsion component in $F_{\perp}$ for separations $z>z_{\mathrm{e}}$. The calculated curves indicate a corrugation $\Delta z=0.6 \AA$ (or 1.25 a.u.) of SFM operating in the constant force mode for $F_{1}$ in the range $\pm 1 \mathrm{nN} /$ atom. The corrugation is expected to be relatively smaller along the edge of the unit cell. The curves in fig. 1c also suggest that in the attractive force range, $\sim-1 \mathrm{nN}<F$ $<0$, the tip may trace an inverted but relatively smaller corrugation if the force gradient is positive. The latter corrugation may be difficult to observe since a conventional SFM cantilever with a soft spring constant $k$ is mechanically unstable in the range where $\partial F_{\perp} / \partial z-k<0$ [3]. Note that the attractive forces acting on the atoms behind the apex add to the force given in fig. 1c when a multilayer tip slab is taken into account. In practice VdW forces neglected, in our treatment, can give an additional attraction.

The above-mentioned scaling expression for $E_{\mathrm{i}}$ leads to $F_{\perp}(z)=-E_{\mathrm{b}} a^{*} \exp \left(-a^{*}\right) / \lambda$. This expression can be useful in obtaining constant force corrugation contours from calculations of $E_{\mathrm{b}}$ at a few mesh points in the surface cell. However, such a fitting procedure is justified only for the perpendicular force in the region of strong attraction.

Measurements of the lateral forces acting on the tip in SFM with perpendicular loading force and scan velocity have revealed atomic-scale vari-

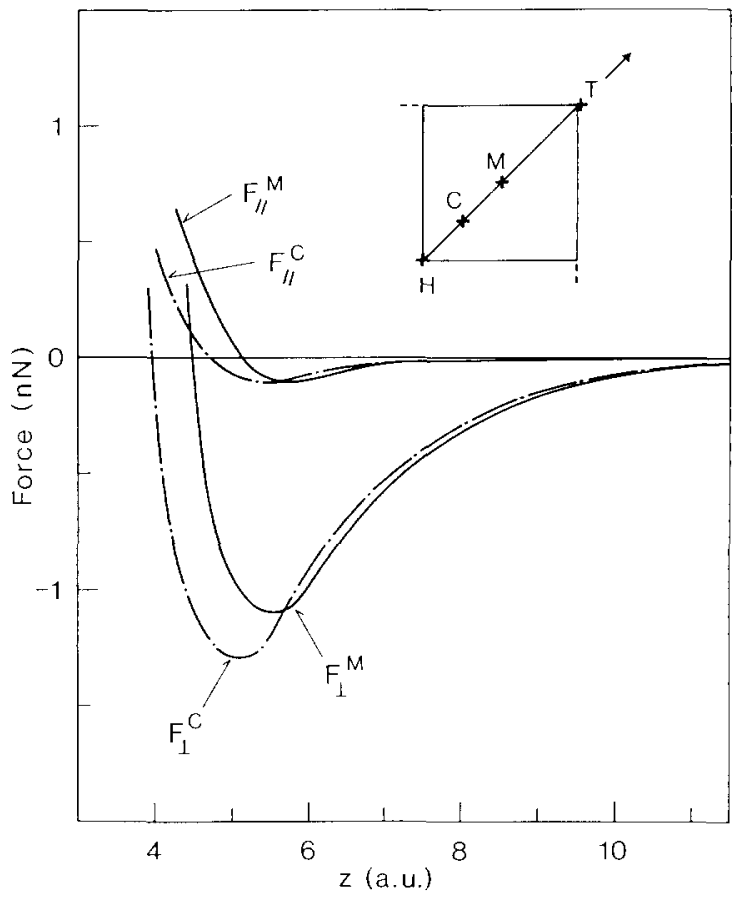

Fig. 2. Perpendicular $F_{L}$ and lateral $F_{\|}$force on the singlelayer "tip slab" versus separation $z$. Forces are calculated at the $\mathrm{C}$ - and $\mathrm{M}$-sites shown in the inset.

ations on graphite [2]. A full account of friction arising in the course of relative stick-slip motion should include the dissipation of energy by phonons and other excitations in both tip and sample, and requires further work. Zhong and Tomanek [16] provided a theoretical estimate of the friction constant $\mu$ from $E_{\mathrm{i}}(z)$ calculated for a commensurate $P t$ monolayer against graphite by assuming that in the slow motion limit the whole potential energy difference between the $\mathrm{T}$ and $\mathrm{H}$-sites is dissipated. The experimental data show an average nonconservative force superposed on a conservative force modulated with the lateral periodicity of the sample surface [2]. An important factor which is ignored in the analysis in ref. [16] is that the strain energy stored in the vicinity of the tip may only be partially released during the stick-slip motion [17]. In the present study we can only calculate the conservative lateral forces $F_{\|}(z)$, as illustrated in fig. 2 for the Mand $\mathrm{C}$-site. For our system they are one order of magnitude smaller than the perpendicular forces 


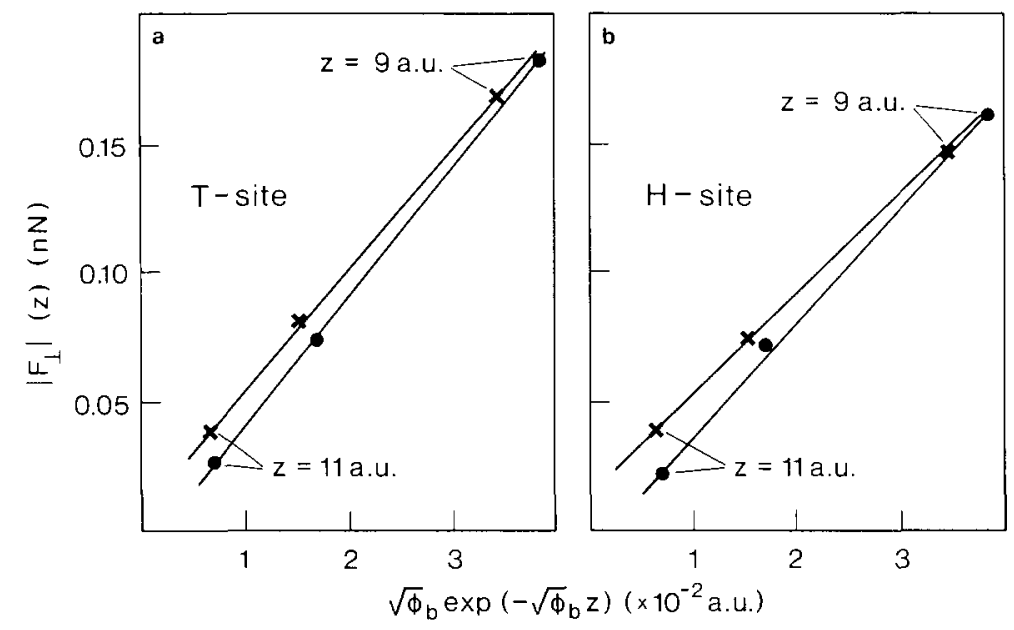

Fig. 3. (a) Perpendicular force $F_{\perp}(z)$ versus the function $\sqrt{\Phi_{b}} \exp \left(-\sqrt{\Phi_{b}} z\right)$ at the top site. Crosses and dots refer to the two-layer and four-layer "tip slab", respectively. (b) Same for the hollow site.

in a wide range of $z$. This can qualitatively be understood as follows: whereas attractive contributions from all neighboring sample atoms tend to add up in $\boldsymbol{F}_{\perp}$, they tend to cancel out in $\boldsymbol{F}_{\|}$. Interestingly, $F_{\|}$can be finite even though $F_{\perp} \simeq 0$, because the zero of $F_{\|}$occurs at a relatively larger $z$.

The fact that the interaction energy is related to the barrier height through the inverse decay constant, $\kappa \simeq \sqrt{\Phi_{b}}$, of the electron density implies a correlation between $F_{\perp}$ and $\Phi_{\mathrm{b}}$ for a given separation $z$. The tunneling barrier with its height and width enters as a crucial parameter in the tunneling conductance $\sigma$. The actual dependence of $\kappa, \Phi_{\mathrm{b}}$ or $\log \sigma$ on $z$ is affected by several factors, such as the lateral modulation of the potential through which electrons tunnel, and its change with decreasing $z$. Earlier we pointed out the reversible modifications of electronic states prior to contact, and correlations between force and barrier height derived thereof $[3,8]$. Experimentally, Dürig et al. [15] drew attention to the correlation between force gradient and tunneling conductance as the tip approaches the sample. More recently, in an effort of presenting a "unified view of STM and SFM", Chen [18] pointed out a simple relation between perpendicular tip force and tunneling conductance. He considered the overlap of the tip and sample wave functions $\left(\psi_{\mathrm{t}}\right.$ and $\left.\psi_{\mathrm{s}}\right)$ near the Fermi level and assumed that the interaction energy is proportional to the splitting of the coupled states through the hopping integral, $\left\langle\psi_{\mathrm{t}}\left|\mathscr{H}_{\mathrm{t}+\mathrm{s}}\right| \psi_{\mathrm{s}}\right\rangle$. Based on this assumption and in line with the work by Flores et al. [19] he argued that $E_{\mathrm{i}}(z)$ is equal to tunneling matrix element $M(z)$, and hence $F_{\perp}(z)=$ $-\partial M / \partial z$, thus leading to $F_{\perp}(z)=\xi \kappa \exp (-\kappa z)$, $\xi$ being proportional to the width of the conduction band. We note that the tunneling matrix element alone can approximately describe the main contribution to $F_{\perp}$ only for $z$ for separation larger than that corresponding to the point of zero force gradient. As pointed out above, in this region the electron-ion interaction and thus the decaying electronic charge density dominate the perpendicular force. For smaller $z$ the barrier collapses and the site-dependence of $F_{\perp}$ becomes pronounced reflecting the ion-ion repulsion. In fig. 3 , we investigate the relation between our calculated $F_{\perp}$ and $\kappa \exp (-\kappa z)$. For the barrier height $\Phi_{\mathrm{b}}$ we took the difference between the maximum of the planar averaged potential at $z / 2$ and the Fermi energy. Despite the fact that the range of variation is limited and that $\Phi_{\mathrm{b}}$ itself is 
calculated between flat metal surfaces using LDA, the linear proportionality depicted by fig. 3 is encouraging and deserves further study.

\section{Conclusions}

We found that the interaction energy and perpendicular forces are strongly site-dependent even for nominally flat $\mathrm{Al}$ surfaces, and that they are well approximated by a universal expression. A significant corrugation is predicted in the constant force mode of SFM operating in the negative force gradient region. Beyond this region at intermediate separations our results show that the perpendicular force and barrier height are interrelated. For the separations considered in this study the lateral tip force is not proportional to the perpendicular one, and generally one order of magnitude smaller.

\section{Acknowledgement}

This work is supported by the Joint Study Agreements between Bilkent University and IBM Almaden Research Center and IBM Zurich Research Laboratory.

\section{References}

[1] G. Binnig, C.F. Quate and Ch. Gerber, Phys. Lett. 56 (1986) 930.

[2] C.M. Mate, G.M. McCleland, R. Erlandson and S. Chiang, Phys. Rev. Lett. 59 (1987) 1942.
[3] S. Ciraci, in: Basic Concepts and Applications of Scanning Tunneling Microscopy and Related Techniques, Vol. E184, Eds. H. Rohrer, N. García and J. Behm (Kluwer, Dordrecht, 1990) p. 119;

S. Ciraci, A. Baratoff and I.P. Batra, Phys. Rev. B 41 (1990) 2763; Phys. Rev. B 42 (1990) 7618.

[4] H.A. Mizes and W.A. Harrison, J. Vac. Sci. Technol. A 6 (1988) 300.

[5] F. Abraham, I.P. Batra and S. Ciraci, Phys. Rev. Lett. 60 (1988) 1314;

F. Abraham and I.P. Batra, Surf. Sci. 209 (1989) L125.

[6] E. Tekman and S. Ciraci, J. Phys. (Condens. Mater) 2 (1991) 2613.

[7] N.D. Lang, Phys. Rev. B 36 (1987) 8173.

[8] S. Ciraci and E. Tekman, Phys. Rev. B 40 (1989) 11969; E. Tekman and S. Ciraci, Phys. Rev. B 43 (1991) 7145; S. Ciraci, Ultramicroscopy $42-44$ (1992) 16.

[9] J.K. Gimzewski and R. Möller, Phys. Rev. B 36 (1987) 1284; N. García, unpublished.

[10] G. Binnig, H. Rohrer, Ch. Gerber and E. Weibel, Phys. Rev. Lett. 49 (1982) 57

[11] D.R. Hamann, M. Schlüter and C. Chiang, Phys. Rev. Lett. 43 (1979) 1494.

[12] D.M. Ceperley and B.J. Alder, Phys. Rev. Lett. 45 (1980) 566.

[13] J. Ihm, A. Zunger and M.L. Cohen, J. Phys. C 12 (1979) 4409;

M.T. Yin and M.L. Cohen, Phys. Rev. B 26 (1982) 3259; I.P. Batra, S. Ciraci, G.P. Srivastava, J.S. Nelson and C.Y. Fong, Phys. Rev. B 34 (1986) 8246.

[14] J. Ferrante and J.R. Smith, Phys. Rev. B 19 (1979) 3911; J.H. Rose, J. Ferrante and J.R. Smith, Phys. Rev. Lett. 47 (1980) 675.

[15] U. Dürig, O. Züger and D.W. Pohl, Phys. Rev. Lett. 65 (1990) 349; U. Dürig and O. Züger, Vacuum 41 (1990) 382.

[16] W. Zhong and D. Tomanek, Phys. Rev. Lett. 64 (1990) 3054.

[17] J.B. Sokoloff, Phys. Rev. Lett. 66 (1991) 965.

[18] F. Flores, A.M. Rodero, E.C. Goldberg and J.C. Duran, Nuovo Cimento 10 (1988) 303. 